\title{
On noncommutative spherically symmetric spaces
}

\author{
Maja Burić1, ${ }^{1,}$ John Madore ${ }^{2, b}$ \\ ${ }^{1}$ Faculty of Physics, University of Belgrade, P.O. Box 44, 11001 Belgrade, Serbia \\ ${ }^{2}$ Laboratoire de Physique Théorique, Orsay 91405, France
}

Received: 21 January 2014 / Accepted: 10 March 2014 / Published online: 27 March 2014

(C) The Author(s) 2014. This article is published with open access at Springerlink.com

\begin{abstract}
Two families of noncommutative extensions are given of a general space-time metric with spherical symmetry, both based on the matrix truncation of the functions on the sphere of symmetry. The first family uses the truncation to foliate space as an infinite set of spheres, and it is of dimension four and necessarily time-dependent; the second can be time-dependent or static, is of dimension five, and uses the truncation to foliate the internal space.
\end{abstract}

\section{Introduction}

Finding an operator description of realistic gravitational configurations is an important task of any theory aiming to describe quantum gravity. Such a description, here referred to as 'quantization', would incorporate at least effectively properties of spacetime beyond the classical regime; it could as well be fundamental. The basic aspect of quantization is representation through operators: concrete representation yields the eigenvalues and the eigenstates of the operators of position, metric and curvature. Therefore, possible outcomes of quantization can be discretization of spacetime, if the spectra of coordinates are discrete, or resolution of singularities, if the classically singular values do not belong to the spectrum of curvature invariants. Another important question, of interest in noncommutative geometry, is whether the algebra of operators $\mathcal{A}$ together with the calculus which supports the given gravitational configuration can in some sense be regarded as a 'noncommutative space', that is, whether notions as connection and metric can be introduced geometrically or are just quantities defined 'externally', as fields with given properties.

In spite of the progress which has been made in recent years in noncommutative geometry and in quantum gravity in general, some important results are still lacking. For physics

\footnotetext{
a e-mail: majab@ipb.ac.rs

b e-mail: madore@th.u-psud.fr
}

the most interesting spacetimes like the Schwarzschild black hole or the Friedmann-Robertson-Walker (FRW) cosmology have not been 'quantized' yet, at least not to common consent. Relevant or 'realistic' models have been obtained only in two dimensions and reduce essentially to the quantum line and the fuzzy sphere. A generalization of the quantum line has been proposed in every dimension [1], and in every even dimension there is a simple smooth noncommutative space with planar symmetry defined by the constant commutators $J_{0}^{\mu \nu}$ between coordinates,

$\left[x^{\mu}, x^{\nu}\right]=i k J^{\mu \nu}=i k J_{0}^{\mu \nu}$.

Usually $J_{0}^{\mu \nu}$ is taken to be nonsingular; the constant $k$ defines the length scale at which the effects of noncommutativity become significant. However, these spaces are not isotropic except in two spatial dimensions while the important classical configurations have spherical symmetry.

Therefore, if we aim to find 'realistic' quantum spacetimes we need to construct four-dimensional spaces with exact or slightly deformed spherical symmetry, and this is the main objective of our paper. The approach we are using is that of noncommutative differential geometry because we believe that one should be able to describe quantum or quantized gravity as in the classical way: through geometry. We shall see that, although the noncommutative spaces which we obtain here do not have exactly the desired classical limits, the formalism which we use seems to be an appropriate tool. We hope that it will be possible to find solutions yet closer to the classical ones by dimensional extension of the algebras here analyzed.

The structure of the paper is as follows. In Sect. 2 we briefly introduce the frame formalism stressing mainly its logic, constraints which it imposes, and symmetries. In Sect. 3 we construct a four-dimensional geometry starting from the momentum space. An analysis of the position space and of its commutative limit shows that the corresponding metric (3.47) is necessarily time-dependent: it describes a 
spherically symmetric non-isotropic cosmology. In Sect. 4 we find a class of static spherically symmetric spaces (4.33), (4.39) and (4.48), (4.49), which include the Schwarzschild black hole, by introducing an additional, Kaluza-Klein-type internal coordinate $\rho$. It is shown that the corresponding phase space has to be extended too, by an external momentum $p_{4}$. Representations of all algebras are also discussed.

In ordinary differential geometry a metric is introduced to measure the distance between points. Since noncommutative geometry is essentially without points the concept might seem in this case rather pointless. It is possible, however, to carry over one of the definitions of a metric which is used in commutative geometry and to give it a meaning in certain noncommutative cases as a measure of distance. In ordinary differential geometry, if $\xi$ and $\eta$ are 1-forms, then the value of the inner product $g(\xi \otimes \eta)$ at a given point does not depend on the values of $\xi$ and $\eta$ at any other point. This condition can be expressed as the bilinearity condition that for any function $f$

$g(f \xi \otimes \eta)=f g(\xi \otimes \eta)=g(\xi \otimes \eta f)=g(\xi \otimes \eta) f$.

We assume that this condition remains valid in the noncommutative case. Without the bilinearity condition it is not possible to distinguish for example in ordinary spacetime a metric which assigns a function to a vector field in such a way that the value at a given point depend only on the vector at that point from one which is some sort of convolution over the entire manifold.

\section{General formalism}

A noncommutative geometry as we define it [2] consists of an associative algebra $\mathcal{A}$ (here referred to as 'noncommutative space') over which we have a differential calculus $\Omega^{*}(\mathcal{A})$. Within this structure we single out a set of $n 1$-forms $\theta^{\alpha}$, a noncommutative extension of the moving frame or $n$-being of classical differential geometry. Conversely one can define the differential calculus to be such that any chosen special set of 1-forms commute with the elements of the algebra

$\left[x^{\mu}, \theta^{\alpha}\right]=0$.

We refer to it as a frame since it seems to be the most natural generalization of the moving frame of Cartan. In fact, the bimodule structure of the 1-forms uniquely determines a differential calculus over the algebra through frame 1-forms $\theta^{\alpha}$ and their dual derivations $e_{\alpha}$,

$\mathrm{d} f=e_{\alpha} f \theta^{\alpha}$
With the frame it is relatively easy at least to first order to calculate the objects of interest in differential geometry: beyond first order more imagination will certainly be necessary.

\subsection{Kinematics}

If instead of the 1 -form $\theta^{\alpha}$ we had an element $f$ of the algebra, then condition (2.1) could be written to first order as

$\left[x^{\mu}, f\right]=i k J^{\mu \nu} \partial_{\nu} f=0$,

and it states that the center of the algebra is trivial. With the frame element, however, we can only state that the condition is of first order in the derivatives. One can think of it then as a constraint. We shall find a second condition,

$e_{\alpha} C^{\alpha}{ }_{\beta \gamma}=0$,

in terms of the Ricci rotation coefficients, which one can think of as a gauge condition.

The derivations $e_{\beta}$ dual to $\theta^{\alpha}, \theta^{\alpha}\left(e_{\beta}\right)=\delta_{\beta}^{\alpha}$, are usually assumed to be inner,

$e_{\alpha} f=\left[p_{\alpha}, f\right], \quad p_{\alpha} \in \mathcal{A}$.

This is the simplest case, and in some important cases [2,3], like matrix spaces, the only choice to define vector fields. Sometimes, however, the condition (2.5) cannot be imposed: in the case of the quantized phase space of quantum mechanics, for example, momenta $p_{\alpha}=-i \hbar \delta_{\alpha}^{\mu} \partial_{\mu}$ do not belong to the algebra of coordinates. When coordinates commute the dimension of the phase space is necessarily twice the dimension of the configuration space. In noncommutative geometry it need not be so: the phase space can be identical to the configuration space, that is, 'half' of its classical analogon. The answer to the question: how many operators generate $\mathcal{A}$, defines as we shall see the initial conditions of the problem which we are trying to solve.

The momenta necessarily satisfy a quadratic relation of the form

$2 P^{\alpha \beta}{ }_{\gamma \delta} p_{\alpha} p_{\beta}-F_{\gamma \delta}^{\beta} p_{\beta}-K_{\beta \gamma}=0$.

This 'structure equation' follows from the conditions which we impose on the differential; it gives the same information about geometry of the space as the set of derivations $e_{\alpha}$ or the set of frame elements $\theta^{\alpha}$. The differential $\mathrm{d} f$ of a function $f \in \mathcal{A}$ is given by

$\mathrm{d} f=\left[p_{\alpha}, f\right] \theta^{\alpha}=\left[p_{\alpha} \theta^{\alpha}, f\right]$ 
when the module of 1-forms is free and the frame is used as basis. We can write to first order

$P_{\gamma \delta}^{\alpha \beta}=\frac{1}{2} \delta_{\gamma \delta}^{\alpha \beta}+i \epsilon Q^{\alpha \beta}{ }_{\gamma \delta}$.

The $i \epsilon$ here is the product of $i k$ and the square $\mu^{2}$ of a macroscopic mass scale parameter $\mu$, which we can relate for example with the Schwarzschild mass $m$ or with the cosmological constant $\Lambda$. As before $k$ defines the scale of noncommutativity through the commutation relations

$\left[x^{\mu}, x^{\nu}\right]=i k J^{\mu \nu}(x)$,

and if we take for example $k \sim l_{P l}^{2}$ and $\mu^{2} \sim \Lambda$, we obtain $\epsilon \sim 10^{-122}$. The coefficients $Q^{\alpha \beta}{ }_{\gamma \delta}, F^{\beta}{ }_{\gamma \delta}, K_{\beta \gamma}$ are antisymmetric in the lower pair of indices, while $Q^{\alpha \beta}{ }_{\gamma \delta}$ is symmetric in the upper pair; they are hermitian elements of the center of the algebra $\mathcal{A}$. Equation (2.6) is equivalent to writing the Ricci rotation coefficients $C_{\alpha \beta}^{\gamma}$,

$\left[e_{\alpha}, e_{\beta}\right]=C_{\alpha \beta}^{\gamma} e_{\gamma}$

as linear expressions in the momenta,

$C_{\alpha \beta}^{\gamma}=F_{\alpha \beta}^{\gamma}-4 i \epsilon p_{\delta} Q^{\gamma \delta}{ }_{\alpha \beta}$.

It can also be considered as the definition of a Poisson structure, to which, as is well known [4-6], one can associate a curvature.

All derivations here are taken in the semi-classical approximation that is to leading order in $\epsilon$. This is in fact the only possibility to do concrete calculations when one is solving a problem like ours: to find an a priori unknown algebra, that is, commutators $J^{\mu \nu}(x)$. Then we have for example

$\left[x^{\mu}, f\right]=i k J^{\mu \nu} \partial_{\nu} f(1+o(\epsilon))$.

It is important to note and easy to see that in this approximation the formalism has diffeomorphism invariance, that is, the commutators transform as tensors. Indeed if we make the change of variables $x^{\prime \mu}=x^{\prime \mu}\left(x^{\rho}\right)$, using (2.12) we obtain

$$
\begin{aligned}
i k J^{\prime \mu \nu} & =\left[x^{\prime \mu}, x^{\prime \nu}\right]=\frac{\partial x^{\prime \mu}}{\partial x^{\rho}} \frac{\partial x^{\prime \nu}}{\partial x^{\sigma}}\left[x^{\rho}, x^{\sigma}\right] \\
& =i k \frac{\partial x^{\prime \mu}}{\partial x^{\rho}} \frac{\partial x^{\prime \nu}}{\partial x^{\sigma}} J^{\rho \sigma} .
\end{aligned}
$$

This means that the diffeomorphism symmetry is present not only in the classical limit but also in the linear order in $k$.

To define and study a noncommutative space $\mathcal{A}$ there are two equivalent paths (the 2-fold way). Starting with a set of vector fields $e_{\alpha}$ on a smooth manifold which satisfy (2.10) one can define the momenta as the solutions to the equations $\left[p_{\alpha}, x^{\mu}\right]=e_{\alpha}^{\mu}$,

which follow from the expression

$\mathrm{d} x^{\mu}=e_{\alpha}^{\mu} \theta^{\alpha}$.

Equation (2.6) then leads to a set of important consistency conditions which one has to solve. Alternatively, one can start from a moving frame $\theta^{\alpha}$ on a classical manifold satisfying

$\mathrm{d} \theta^{\gamma}=-\frac{1}{2} C^{\gamma}{ }_{\alpha \beta} \theta^{\alpha} \theta^{\beta}$,

and search for an analogous noncommutative frame, extending thus $\Omega^{*}(\mathcal{C})$ to $\Omega^{*}(\mathcal{A})$. In both cases one obtains the essential ingredients to construct a noncommutative geometry, an algebra, and a compatible differential calculus. We find, however, that there are restrictions on the ingredients which arise from the Jacobi identities.

\subsection{Dynamics}

The commutator (2.9) must satisfy the Jacobi identities, which can be written in the form

$A_{J \rho}=\epsilon_{\rho \lambda \mu \nu}\left[x^{\lambda}, J^{\mu \nu}\right]=0$,

showing vanishing of an anomaly. We shall accept the assumption that this condition is the only obstruction to the associativity of the product. There are also Leibniz rules, obtained by replacing one position generator $x^{\lambda}$ by a momentum $p_{\alpha}$. As we are restricting our attention to the case when the momenta depend on the coordinates, the additional Jacobi identities follow from (2.17). The Leibniz rules can be written in the form

$\mathrm{d}\left(\left[x^{\mu}, x^{\nu}\right]-i k J^{\mu \nu}\right)=0$.

It is easily seen that the analogous compatibility conditions for (2.9),

$\mathrm{d}\left(\left[x^{\mu}, \theta^{\alpha}\right]\right)=0$,

are equivalent in fact to the quadratic relation (2.6) for the momenta.

\section{Real-space foliation}

The simplest spherically symmetric noncommutative space is the fuzzy sphere [7,8]. Anticipating that isotropic spaces in higher dimensions contain it as a subspace, we will review its basic properties. Two angular variables of the polar coordinate system on the fuzzy sphere are described by three 
Cartesian coordinates which satisfy commutation relations of the algebra of rotations. The Lie algebra $\mathrm{SO}_{3}$ has irreducible representations by three $n \times n$ complex matrices $J^{a} \in M_{n}$, which we normalize such that

$\left[J^{a}, J^{b}\right]=i \epsilon^{a b c} J_{c}, \quad J^{2}=\frac{1}{4}\left(n^{2}-1\right)$.

Let $x^{a}, a=1,2,3$ be the quantized coordinates. Quantization of the sphere is defined by the relation

$x^{a}=\frac{k}{r} J^{a}$,

which is an analog of the Bohr quantization and postulates that the area of the quantum sphere contains an integer number of elementary cells of area $k$. It also defines the radius $r$ since from the Casimir relation of $\mathrm{So}_{3}$ for large $n$ we obtain

$r^{2}=\delta_{a b} x^{a} x^{b}=\frac{k n}{2}$

The commutator of the coordinates is then given by

$\left[x^{a}, x^{b}\right]=i k C^{a b}{ }_{c} x^{c}=\frac{i k}{r} \epsilon^{a b c} x_{c}$.

Equation (3.3) suggests that the three-dimensional space in polar coordinates is, or can be represented as, a direct sum

$\mathcal{A}_{3}=\bigoplus_{n} M_{n}$

of all irreducible representations of $\mathrm{So}_{3}$ : this model is called the 'onion model'. As we need a four-dimensional manifold we shall not use it; simple extensions by one algebra generator do not have the desired properties and limits too $[9,10]$. Therefore we add the radius and the time as coordinates independent on $x^{a}$. That is, we consider the spacetime $\mathcal{A}=\mathcal{A}^{\prime} \otimes \mathcal{A}^{\prime \prime}$ to be the tensor product of the fuzzysphere algebra $\mathcal{A}^{\prime}$ and an algebra $\mathcal{A}^{\prime \prime}$ generated by $r$ and $t$.

The momenta on the fuzzy sphere can be identified with the coordinates,

$i k p_{a}=\delta_{a b} x^{b}$

We have therefore

$\left[p_{a}, p_{b}\right]=C_{a b}{ }^{c} p_{c}, \quad C_{a b c}=\frac{1}{r} \epsilon_{a b c}$

and if we let $\pi_{b}^{a}=\delta_{b}^{a}-\frac{1}{r^{2}} x^{a} x_{b}$ be the radial projection onto the sphere, we obtain from definition (2.7) the expression for the differentials $\mathrm{d} x^{a}$,

$\mathrm{d} x^{a}=\frac{1}{r} \epsilon_{b c}^{a} x^{b} \theta^{c}$, and inversely

$\pi_{b}^{a} \theta^{b}=-\frac{1}{r} \epsilon_{b c}^{a} x^{b} \mathrm{~d} x^{c}+\frac{i k}{r^{2}} \delta_{c}^{a} \mathrm{~d} x^{c}$.

In the leading order in $k$ we can write

$\mathrm{d} x^{a}=C^{a}{ }_{b c} x^{b} \pi_{d}^{c} \theta^{d}, \quad \pi_{b}^{a} \theta^{b}=-C^{a}{ }_{b c} x^{b} \mathrm{~d} x^{c}$.

\subsection{The momentum algebra}

Since the information as regards symmetries is contained in the frame derivatives $e_{\alpha}$ that is in $p_{\alpha}$, the momentum algebra ${ }^{1}$ is the best starting point to search for $\mathcal{A}$. It has in addition a fixed quadratic form which reduces the number of possible Ansätze. We investigate then the extensions of the $s_{3} o_{3}$ algebra by the two operators $p_{0}$ and $p_{4}$ which we introduce in place of $r$ and $t$ as generators of $\mathcal{A}^{\prime \prime}$. We start with an algebra of a general form

$\left[p_{a}, p_{b}\right]=\frac{i \epsilon}{2} \epsilon_{a b}^{c}\left(\Pi p_{c}+p_{c} \Pi\right)$,

$\left[p_{0}, p_{c}\right]=\frac{i \epsilon}{2}\left(\pi_{0} p_{c}+p_{c} \pi_{0}\right)$,

$\left[p_{4}, p_{c}\right]=\frac{i \epsilon}{2}\left(\pi_{4} p_{c}+p_{c} \pi_{4}\right)$,

$\left[p_{0}, p_{4}\right]=i \epsilon \Xi$,

$\left[\Xi, p_{c}\right]=\frac{i \epsilon}{2}\left(\Upsilon p_{c}+p_{c} \Upsilon\right)$

which manifestly respects rotational symmetry. In accordance with the requirement that the algebra be quadratic we write

$\Pi=a+b p_{0}+c p_{4}$,

$\pi_{0}=a_{0}+b_{0} p_{0}+c_{0} p_{4}$

$\pi_{4}=a_{4}+b_{4} p_{0}+c_{4} p_{4}$.

The $a_{k}, b_{k}$, and $c_{k}$ are constants, while $\Xi$ is a general quadratic polynomial in $p_{0}$ and $p_{4}$. When we impose Jacobi identities on (3.11-3.15) we obtain various restrictions. For example we find

$\Upsilon=\left(b_{0}+c_{4}\right) \Xi, \quad c \Xi=\frac{1}{2}\left(\pi_{0} \Pi+\Pi \pi_{0}\right)$,

$b \Xi=-\frac{1}{2}\left(\pi_{4} \Pi+\Pi \pi_{4}\right)$,

and from these relations we conclude that $\pi_{0}$ and $\pi_{4}$ are mutually proportional,

$b \pi_{0}+c \pi_{4}=0$.

${ }^{1}$ We refer to the algebras generated by the coordinates and the momenta, respectively, as the position algebra and the momentum algebra although we assume in this section they are one and the same algebra. 
The constants are also not independent: we have

$b a_{0}+c a_{4}=0, \quad b b_{0}+c b_{4}=0, \quad b c_{0}+c c_{4}=0$.

The most important implication of the Jacobi constraints is that there always exists a linear combination of $p_{0}$ and $p_{4}$ which commutes with $p_{a}$ : it is in fact equal to $\Pi$. Using $\Pi$, the momentum algebra simplifies to

$\left[p_{a}, p_{b}\right]=i \epsilon \epsilon_{a b}{ }^{c} \Pi p_{c}, \quad\left[\Pi, p_{c}\right]=0$,

$\left[\pi_{4}, p_{c}\right]=\frac{i \epsilon}{2} c\left(\pi_{4} p_{c}+p_{c} \pi_{4}\right)$,

$\left[\pi_{4}, \Pi\right]=\frac{i \epsilon}{2} c\left(\pi_{4} \Pi+\Pi \pi_{4}\right)$

where we substituted

$\frac{b c_{0}}{c}-b_{0} \rightarrow c$

We adopt here the convention that the momenta are antihermitian operators.

It is easy to rewrite the algebra (3.22) in the tensor-product form by introducing the hermitian frame components $\zeta^{a}$ of a vector (which we will later use as coordinate),

$\zeta_{a}=\Pi^{-1} p_{a}$.

We have

$\left[\zeta_{a}, \zeta_{b}\right]=i \epsilon \epsilon_{a b}{ }^{c} \zeta_{c}, \quad\left[\Pi, \zeta_{a}\right]=0$,

$\left[\pi_{4}, \zeta_{a}\right]=0, \quad\left[\Pi, \pi_{4}\right]=\frac{i \epsilon c}{2}\left(\Pi \pi_{4}+\pi_{4} \Pi\right)$.

We see that $\mathcal{A}$ indeed contains an so $_{3}$ as a subalgebra; $\mathcal{A}$ is a tensor product of the $\mathrm{So}_{3}$ generated by three $\zeta_{a}$ and a quadratic $\mathcal{A}^{\prime \prime}$ defined by the relation

$\Pi \pi_{4}=q \pi_{4} \Pi$,

with

$q=\frac{2+i \epsilon c}{2-i \epsilon c}$

However, differential calculus defined by $\left(p_{a}, \Pi, \pi_{4}\right)$ is not a tensor-product calculus because the momenta do not belong to the factor algebras. This property is desired as otherwise for the metric we would obtain a simple product metric.

We mentioned already that within the frame formalism the momentum algebra determines space-time geometry. Of course there is a freedom in the choice of the linear connection, which then uniquely gives the torsion and the curvature; these expressions one can show are generically quadratic in the momenta, $[2,11]$. But momenta are a priori unknown functions of coordinates. Therefore although our model is essentially fixed, to understand it in more detail and to find its classical limit we need to determine coordinates.

\subsection{The position algebra}

The functional dependence of $p_{\alpha}$ on $x^{\mu}$ is not unique, but as everything else it is constrained by the algebraic structure of $\mathcal{A}$ and the required properties of the commutative limit. In the present case there is only one spatial 3-vector in the algebra so it is natural to assume that coordinates $x^{a}$ are proportional to it. In fact, we already introduced the hermitian $\zeta^{a}, p_{a}=$ $\Pi \zeta_{a}$, as generators which provide the tensor-product form of the algebra (3.25). We can thus choose $\zeta^{a}$ as coordinates, $x^{a}=\zeta^{a}$; one can see easily from (3.43) that the choice of the proportionality factor does not influence the form of the angular part of the line element. Of course, only two of the $\zeta^{a}$ are independent because $\zeta^{2}=\zeta^{a} \zeta_{a}$ is the Casimir of $\mathcal{A}$. The operators $\Pi$ and $\pi_{4}$ have to be mutually independent functions of $r$ and $t$. An almost obvious Ansatz for these functions is

$i \epsilon \Pi=F(r)=\frac{1}{r}, \quad i \epsilon \pi_{4}=G(t)$.

The coordinate components of the (inverse) metric can be found from the relation

$g^{\mu \nu}=e_{\alpha}^{\mu} e_{\beta}^{\nu} \eta^{\alpha \beta}$

where the frame components $e_{\alpha}^{\mu}$ are given by $e_{\alpha}^{\mu}(x)=$ $\left[p_{\alpha}, x^{\mu}\right]$. One can also use the inverse $\theta_{\mu}^{\alpha}$ of $e_{\alpha}^{\mu}$,

$g_{\mu \nu}=\theta_{\mu}^{\alpha} \theta_{v}^{\beta} \eta_{\alpha \beta}$

The two given expressions are equivalent, within the precision we are working with, that is, as regards the operatorordering ambiguities. We can also write the line element as

$\mathrm{d} s^{2}=\eta_{\alpha \beta} \theta^{\alpha} \otimes \theta^{\beta}$,

and then express the frame 1-forms through the differentials, $\theta^{\alpha}=\theta_{\mu}^{\alpha}(x) \mathrm{d} x^{\mu}$, to obtain $g_{\mu \nu}$. The constant frame metric $g_{\alpha \beta}=\eta_{\alpha \beta}$ here has the signature $(-++++)$.

The commutator of $r$ and $t$,

$[r, t]=i k J(t, r)$

can be obtained from compatibility of (3.22) with (3.32). Using it we find easily

$J=-c \mu^{2} r \frac{G}{\dot{G}}$. 
For the differentials of the coordinates we have

$$
\begin{aligned}
\mathrm{d} \zeta^{i} & =\left[p_{b}, \zeta^{i}\right] \theta^{b}+\left[\Pi, \zeta^{i}\right] \theta^{0}+\left[\pi_{4}, \zeta^{i}\right] \theta^{4} \\
& =\frac{1}{r} \delta^{i a} \epsilon_{a b c} \zeta^{b} \theta^{c} \\
\mathrm{~d} r & =\operatorname{cr} G \theta^{4} \\
\mathrm{~d} t & =\frac{c}{r} \frac{G}{\dot{G}}\left(\delta_{i a} \zeta^{i} \theta^{a}+\theta^{0}\right)
\end{aligned}
$$

where we introduced $\zeta^{i}=\delta_{a}^{i} \zeta^{a}$. From these expressions we obtain the frame components

$e_{c}^{i}=\frac{1}{r} \delta^{i a} \epsilon_{a b c} \zeta^{b}, \quad e_{4}^{4}=c r G, \quad e_{a}^{0}=\frac{c}{r} \frac{G}{\dot{G}} \zeta_{a} \quad e_{0}^{0}=\frac{c}{r} \frac{G}{\dot{G}}$.

We can calculate the metric by applying (3.30); the nonzero elements are

$g^{i j}=e_{a}^{i} e_{b}^{j} \delta^{a b}=\frac{1}{r^{2}}\left(\delta^{i j} \delta^{b d}-\delta^{i d} \delta^{j b}\right) \zeta_{b} \zeta_{d}=\frac{1}{r^{2}} \delta_{a}^{i} \delta_{b}^{j} \zeta^{2} \pi^{a b}$,

$g^{44}=(c r G)^{2}$,

$g^{00}=-\left(1-\zeta^{2}\right)\left(\frac{c}{r} \frac{G}{\dot{G}}\right)^{2}$,

$g^{0 i}=i k \frac{c}{r^{2}} \frac{G}{\dot{G}} \zeta^{i}$

We see immediately that in the classical limit $k \rightarrow 0$ offdiagonal terms $g^{0 i}$ vanish. Further, since we can assume that the Casimir $\zeta^{2}=\frac{1}{4} \epsilon^{2}\left(n^{2}-1\right)$ is small, we have $1-\zeta^{2}>0$. The angular part of the metric is, as expected, proportional to the projector on the sphere $\pi^{a b}$,

$\pi^{a b}=\delta^{a b}-\frac{\zeta^{a} \zeta^{b}}{\zeta^{2}}$

The prefactor in (3.38) is also correct: the inverse of $g^{i j}$ is on the sphere given by

$g_{i j}=\frac{r^{2}}{\zeta^{2}} \delta_{i}^{a} \delta_{j}^{b} \pi_{a b}$

and therefore the angular part of the line element is

$\mathrm{d} s_{\Omega}^{2}=g_{a b} d \zeta^{a} d \zeta^{b}=r^{2} d \Omega$.

In the classical limit we obtain

$\mathrm{d} s^{2}=-\frac{r^{2}}{c^{2}\left(1-\zeta^{2}\right)} \frac{\dot{G}^{2}}{G^{2}} \mathrm{~d} t^{2}+\frac{1}{c^{2} r^{2} G^{2}} \mathrm{~d} r^{2}+r^{2} \mathrm{~d} \Omega$.

We have a time-dependent metric. Namely, we cannot assume that $G(t)=G_{0}$, a constant, because the momenta $\Pi$ and $\pi_{4}$ have to be functionally independent. Introducing instead of $t$ a new variable $\tau$,

$\frac{\dot{G}}{G} \mathrm{~d} t=-\lambda \mathrm{d} \tau, \quad G=e^{-\lambda \tau}$

we can simplify the line element to

$\mathrm{d} s^{2}=-\frac{\lambda^{2} r^{2}}{c^{2}\left(1-\zeta^{2}\right)} \mathrm{d} \tau^{2}+\frac{1}{c^{2} r^{2}} e^{2 \lambda \tau} \mathrm{d} r^{2}+r^{2} \mathrm{~d} \Omega$,

with the curvature scalar $R$

$R=\frac{2\left(1+c^{2}-c^{2} \zeta^{2}\right)}{r^{2}}-12 c^{2} e^{-2 \lambda \tau}$

This metric, though spherically symmetric is obviously not homogeneous. The universe expands differently in different directions: if we define the Hubble parameter $H$ as the rate of expansion in the radial direction we find that $H=\lambda$. The $\lambda$ on the other hand is related to the noncommutativity of space-time,

$[r, \tau]=-\frac{i k c}{\lambda} r$

An interesting change of variables, $\eta=G(t), \chi=\frac{1}{r}$, transforms the line element (3.47) to an almost conformally flat form,

$\mathrm{d} s^{2}=\frac{1}{c^{2} \eta^{2} \chi^{2}}\left(-\frac{1}{1-\zeta^{2}} \mathrm{~d} \eta^{2}+\mathrm{d} \chi^{2}+c^{2} \eta^{2} \mathrm{~d} \Omega\right)$.

Since the Ansatz (3.28) which we used does not give a static metric which could be interpreted as an extension of the Schwarzschild black hole, perhaps it is possible to modify it to obtain the extension of the FRW cosmology? Then instead of (3.44) we would need the angular line element $\mathrm{d} s_{\Omega}^{2}=$ $a^{2}(t) r^{2} \mathrm{~d} \Omega$. This corresponds presumably to a more general dependence,

$i \epsilon \Pi=F(r) N(t), \quad i \epsilon \pi_{4}=L(r) G(t)$.

Let us analyse this possibility leaving the commutator Ansatz the same; see (3.32). A comparison of (3.51) with (3.22) gives the compatibility equation

$-\frac{\dot{N}}{N} \frac{L^{\prime}}{L}+\frac{\dot{G}}{G} \frac{F^{\prime}}{F}=\frac{c \mu^{2}}{I_{0} I_{4}}$

As the variables $r$ and $t$ are separated it is not difficult to find a solution to the last equation. We have

$-\frac{F^{\prime}}{F}=\frac{\alpha}{I_{4}}, \quad-\frac{L^{\prime}}{L}=\frac{\beta}{I_{4}}, \quad \frac{\dot{N}}{N}=\frac{\gamma}{I_{0}}, \quad \frac{\dot{G}}{G}=\frac{\lambda}{I_{0}}$, 
where the constants $\alpha, \beta, \gamma$, and $\lambda$ satisfy

$\beta \gamma-\alpha \lambda=c \mu^{2} \neq 0$

The change the variables to

$\rho(r)=-\int \frac{\mathrm{d} r}{I_{4}(r)}, \quad \tau(t)=\int \frac{\mathrm{d} t}{I_{0}(t)}$,

drastically simplifies all equations and we can solve (4.17):

$F=e^{\alpha \rho}, \quad L=e^{\beta \rho}, \quad N=e^{\gamma \tau}, \quad G=e^{\lambda \tau}$.

Now it is possible to calculate the metric. From the differentials

$\mathrm{d} \zeta^{a}=F N \epsilon_{a b c} \zeta^{b} \theta^{c}$,

$\mathrm{d} r=-J\left(F \dot{N} \zeta_{b} \theta^{b}+F \dot{N} \theta^{0}+L \dot{G} \theta^{4}\right)$,

$\mathrm{d} t=J\left(F^{\prime} N \zeta_{b} \theta^{b}+F^{\prime} N \theta^{0}+L^{\prime} G \theta^{4}\right)$,

we obtain the nonvanishing components of the frame,

$e_{c}^{i}=F N \delta^{i a} \epsilon_{a b c} \zeta^{b}$,

$e_{a}^{4}=-J F \dot{N} \zeta_{a}, \quad e_{0}^{4}=-J F \dot{N}, \quad e_{4}^{4}=-J L \dot{G}$,

$e_{a}^{0}=J F^{\prime} N \zeta_{a}, \quad e_{0}^{0}=J F^{\prime} N, \quad e_{4}^{0}=J L^{\prime} G$.

Using (3.30) we find

$$
\begin{aligned}
& g^{i j}=(F N)^{2} \zeta^{2} \delta_{a}^{i} \delta_{b}^{j} \pi^{a b}, \\
& g^{44}=J^{2}\left(-(F \dot{N})^{2}\left(1-\zeta^{2}\right)+(L \dot{G})^{2}\right), \\
& g^{00}=J^{2}\left(-\left(F^{\prime} N\right)^{2}\left(1-\zeta^{2}\right)+\left(L^{\prime} G\right)^{2}\right), \\
& g^{04}=J^{2}\left(F^{\prime} N F \dot{N}\left(1-\zeta^{2}\right)-L^{\prime} G L \dot{G}\right), \\
& g^{0 i}=-i k J F F^{\prime} N^{2} \zeta^{i}, \\
& g^{4 i}=i k J F^{2} N \dot{N} \zeta^{i} .
\end{aligned}
$$

Again the off-diagonal components $g^{0 i}$ and $g^{4 i}$ vanish in the commutative limit and the angular part is proportional to the projector on the sphere: the corresponding classical metric is spherically symmetric, non-static, and block-diagonal. Inverting the angular part we get

$g_{a b}=(F N)^{-2} \zeta^{-2} \pi_{a b}$,

that is,

$\mathrm{d} s_{\Omega}^{2}=g_{a b} d \zeta^{a} d \zeta^{b}=(F N)^{-2} d \Omega$.
The $(r, t)$ part on the other hand gives

$g_{00}=\frac{1}{c^{2} I_{0}^{2}}\left(\frac{\gamma^{2}}{(L G)^{2}}-\frac{\lambda^{2}}{\left(1-\zeta^{2}\right)(F N)^{2}}\right)$,

$g_{44}=\frac{1}{c^{2} I_{4}^{2}}\left(\frac{\alpha^{2}}{(L G)^{2}}-\frac{\beta^{2}}{\left(1-\zeta^{2}\right)(F N)^{2}}\right)$,

$g_{04}=\frac{1}{c^{2} I_{0} I_{4}}\left(\frac{\alpha \gamma}{(L G)^{2}}-\frac{\beta \lambda}{\left(1-\zeta^{2}\right)(F N)^{2}}\right)$.

One can further simplify using the variables $\rho$ and $\tau$ and obtain

$$
\begin{aligned}
\mathrm{d} s_{\rho}^{2}= & -\frac{1}{c^{2}\left(1-\zeta^{2}\right)(F N)^{2}}(\lambda d \tau+\beta d \rho)^{2} \\
& +\frac{1}{c^{2}(L G)^{2}}(\gamma d \tau+\alpha d \rho)^{2} .
\end{aligned}
$$

Having the line element written as this, it is easy to recognize that the change of coordinates

$F N \rightarrow \frac{1}{r}, \quad L G \rightarrow e^{-\lambda \tau}$

gives back the metric (3.47). This is a manifestation of diffeomorphism invariance of the formalism, and it shows that the noncommutative space which we obtained is in fact unique.

\subsection{A representation}

The coordinates of the solution (3.28) satisfy the commutation relation

$e^{\lambda \tau} r=q^{-1} r e^{\lambda \tau}$.

Parameter $q$ defined in (3.27) is unitary because the constant $c$ is real, while $r$ and $\tau$ are hermitian operators. Equation (3.74) can be rewritten as

$e^{\lambda \tau} r e^{-\lambda \tau}=r+\lambda[\tau, r]+\frac{\lambda^{2}}{2 !}[\tau,[\tau, r]]+\cdots=q^{-1} r$,

and we see that it holds for

$[\tau, r]=i k r$

with $\tan (k \lambda / 2)=\epsilon c / 2$ : the algebra is formally equivalent to the Heisenberg algebra.

The spectrum of $\tau$ is the real line and that of $r$ the positive real line. A representation on the Hilbert space of square integrable functions of one variable $s$ is given by

$\tau=\frac{i k}{4}\left(s \frac{\mathrm{d}}{\mathrm{d} s}+\frac{\mathrm{d}}{\mathrm{d} s} s\right), \quad r=s^{2}$, 
where $\tau$ is the dilatator, or by

$\tau=i k \frac{\mathrm{d}}{\mathrm{d} s}, \quad r=e^{s}$

We have here an indication of the importance of the calculi in the description of the geometries: the differential calculus usually introduced on a space described by the Heisenberg algebra is flat, while here the subspace $(r, \tau)$ has a constant negative curvature, [12]. One can find even an example of an algebra over which there are two different calculi with geometries having as the commutative limit two different topologies.

\section{Internal-space foliation}

We have seen that in the previous section geometry of the space-time was fully determined by momenta and by their commutators. Such a situation is typical when we apply the noncommutative frame formalism in its 'minimal' version, that is, when all $p_{\alpha} \in \mathcal{A}$. A close relation between the algebraic and the geometric structures is, however, a general feature of any noncommutative geometry, also in cases when we need to extend the space-time in order to obtain the appropriate phase space or the prescribed symmetries.

We shall attempt now to find a static spherically symmetric metric starting from the algebra of coordinates. This means that we will first make an Ansatz for the position commutators and for the frame, and then analyze the implied consistency relations. This approach is perhaps more intuitive and seems easier because the position algebra is not restricted in its form like the momentum algebra. However, the overall number of equations remains the same if we constrain the momenta to belong to $\mathcal{A}$ : a real advance comes if we allow for some of the derivations to be external. As a result, we shall find a way to extend $\mathcal{A}$ and to obtain static solutions.

\subsection{The position algebra}

Assume that $\mathcal{A}$ is generated by operators $x^{\mu}=\left(\xi^{a}, \rho, r, t\right)$ with for some large $n$

$\xi^{a}=\frac{2}{n} J^{a}$

Variable $\rho$ is a fifth generator which we can consider as a Kaluza-Klein extension. We expect a general spherically symmetric solution to depend on $\rho, r$, and $t$, but we shall restrict our attention to the static case.

The multiplication table is

$$
\left[\xi^{a}, \xi^{b}\right]=\frac{2 i}{n} \epsilon^{a b c} \xi_{c}, \quad\left[\xi^{a}, \rho\right]=\left[\xi^{a}, r\right]=\left[\xi^{a}, t\right]=0,
$$

$$
\begin{aligned}
& {[\rho, t]=i k J^{0} \rho, \quad[r, t]=i k J,} \\
& {[\rho, r]=i k J^{4} \rho,}
\end{aligned}
$$

and to ensure spherical symmetry we assume that $J=$ $J(\rho, r, t), J^{0}=J^{0}(\rho, r, t), J^{4}=J^{4}(\rho, r, t)$. The position algebra (4.2-4.3) is restricted by Jacobi identities. The nontrivial one is

$[\rho,[r, t]]+[r,[t, \rho]]+[t,[\rho, r]]=0$,

and it gives to first order the equation

$J^{0} \dot{J}-J \dot{J}^{0}+J^{4} J^{\prime}-J J^{4 \prime}+J^{4} \rho \partial_{\rho} J^{0}-J^{0} \rho \partial_{\rho} J^{4}=0$,

or if we introduce $\gamma_{0}$ and $\gamma_{4}$ as $J^{0}=J \gamma_{0}, J^{4}=J \gamma_{4}$,

$\dot{\gamma}_{0}+\gamma_{4}^{\prime}+\gamma_{0} \rho \partial_{\rho} \gamma_{4}-\gamma_{4} \rho \partial_{\rho} \gamma_{0}=0$.

As a convenient solution we can choose

$J^{4}=0, \quad \gamma_{4}=0$,

which implies

$\dot{\gamma}_{0}=0$.

The last equation is identically fulfilled in the static case.

For comparison with the previous section we introduce $x^{a}=\rho \xi^{a}, x^{a} x^{b} \delta_{a b}=\rho^{2}$ and the intermediate variable $L=2 \rho^{2} / n k$. Then we have

$\left[x^{a}, x^{b}\right]=\frac{i k}{\rho} L \epsilon^{a b}{ }_{c} x^{c}$

\subsection{The frame}

As the angular part of the frame we choose the Mauer-Cartan frame of the group multiplied by a function $h$ to account for the change in volume of the 3-sphere as we move along the radial; the radial and the time-like components of the frame are, we suppose, diagonal. We obtain for the frame in five dimensions

$$
\begin{aligned}
\theta^{a}= & -h \rho^{-1} \epsilon^{a}{ }_{b c} x^{b} \mathrm{~d} x^{c} & & \\
& +\rho^{-2} x_{b} \theta^{b} x^{a}, & & \mathrm{~d} x^{a}=(h \rho)^{-1} \epsilon^{a}{ }_{b c} x^{b} \theta^{c}, \\
\theta^{4}= & g \mathrm{~d} r, & & \mathrm{~d} r=g^{-1} \theta^{4}, \\
\theta^{0}= & f \mathrm{~d} t, & & \mathrm{~d} t=f^{-1} \theta^{0} .
\end{aligned}
$$

The $f, g$, and $h$ are functions of $\rho, r$, and $t$. As on the fuzzy sphere this Ansatz gives

$\rho \mathrm{d} \rho+\mathrm{d} \rho \rho=0$

which is unusual because it implies $\mathrm{d} \rho=0$ in linear order even though $\rho$ is not in the center. In particular, $d \rho=0$ in 
the classical limit. This is a specific feature of our calculus (4.11) and is a consequence of the fact that the algebra of coordinates, as we shall see, is different from the algebra of momenta.

To be consistent, the differential calculus (4.11) has to be compatible with the algebra (4.2-4.3), and this compatibility is expressed by (2.18). To leading order the right-hand side of these equations is given by

$\mathrm{d} J^{\mu \nu}=\partial_{\rho} J^{\mu \nu} \mathrm{d} x^{\rho}=\partial_{\rho} J^{\mu \nu} e_{\alpha}^{\rho} \theta^{\alpha}$,

while to calculate the left-hand side we use the property that the elements of the frame basis commute with $\mathcal{A}$,

$\left[\mathrm{d} x^{\mu}, x^{\nu}\right]=\left[e_{\alpha}^{\mu} \theta^{\alpha}, x^{\nu}\right]=\left[e_{\alpha}^{\mu}, x^{\nu}\right] \theta^{\alpha}$.

An analysis of all constraints which follow from these requirement yields:

$\dot{L}=0, \quad L^{\prime}=0$,

$\dot{J}^{4}=0, \quad J^{0 \prime}=0, \quad h^{\prime} J^{4}+\dot{h} J^{0}=0$

and

$J^{4 \prime}+g^{-1}\left(g^{\prime} J^{4}+\dot{g} J^{0}\right)=0, \quad\left(h+\rho \partial_{\rho} h\right) J^{4}-\dot{h} J=0$,

$J^{0 \prime}+f^{-1}\left(f^{\prime} J^{4}+\dot{f} J^{0}\right)=0, \quad\left(h+\rho \partial_{\rho} h\right) J^{0}+h^{\prime} J=0$,

$\dot{J}+f^{-1}\left(\dot{f} J-\rho \partial_{\rho} J^{4}\right)=0, \quad J^{\prime}+g^{-1}\left(g^{\prime} J+\rho \partial_{\rho} J^{0}\right)=0$.

We must impose additional relations, which ensure that the exterior multiplication of two frame 1 -forms is well defined. These relations follow from the definition of the wedge product [2],

$\theta^{\alpha} \theta^{\beta}=P_{\gamma \delta}^{\alpha \beta} \theta^{\gamma} \theta^{\delta}$.

In lowest order we obtain

$\left[\theta^{\alpha}, \theta^{\beta}\right]=i k \mu^{2} Q^{\alpha \beta}{ }_{\gamma \delta} \theta^{\gamma} \theta^{\delta}$,

where $Q^{\alpha \beta}{ }_{\gamma \delta}$ are the constants introduced in (2.8). In our case, (4.18) becomes the following equations:

$\left[\theta^{a}, \theta^{b}\right]=0$,

$\left[\theta^{0}, \theta^{0}\right]=i k f g^{-1} \partial_{r}\left(f^{-1} f^{\prime} J+\rho f^{-1} \partial_{\rho} f J^{0}\right) \theta^{4} \theta^{0}$,

$\left[\theta^{4}, \theta^{4}\right]=-i k g f^{-1} \partial_{t}\left(g^{-1} \dot{g} J-\rho g^{-1} \partial_{\rho} g J^{4}\right) \theta^{0} \theta^{4}$,

$\left[\theta^{0}, \theta^{4}\right]+(f g)^{-1}[f, g] \theta^{4} \theta^{0}$

$=i k \partial_{t}\left(g^{-1} g^{\prime} J+\rho g^{-1} \partial_{\rho} g J^{0}\right) \theta^{0} \theta^{4}$,

$=-i k \partial_{r}\left(f^{-1} \dot{f} J-\rho f^{-1} \partial_{\rho} f J^{4}\right) \theta^{4} \theta^{0}$.
There is one remaining relation for $\left[\theta^{a}, \theta^{0}\right]$, which can be obtained from

$$
\begin{aligned}
{\left[\mathrm{d} x^{a}, \mathrm{~d} t\right]=} & \frac{1}{\rho h f} \epsilon_{b c}^{a} x^{b}\left[\theta^{c}, \theta^{0}\right] \\
& +\frac{1}{\rho h^{2} f^{2}} \epsilon_{b c}^{a} x^{b}[h, f] \theta^{c} \theta^{0} \\
= & -\frac{i k}{\rho h f} \epsilon_{b c}^{a} x^{b} J f^{-1}\left(\gamma_{4} f^{\prime}+\gamma_{0} \dot{f}\right) \theta^{c} \theta^{0} \\
& -\frac{i \hbar}{f g} x^{a} \partial_{r}\left(J f^{-1}\left(\gamma_{4} f^{\prime}+\gamma_{0} \dot{f}\right)\right) \theta^{4} \theta^{0} \\
= & \frac{i \hbar}{\rho h f} \epsilon_{b c}^{a} x^{b} \partial_{t}\left(J h^{-1}\left(h^{\prime}+\gamma_{0} \rho \partial_{\rho} h\right)\right) \theta^{0} \theta^{c} \\
& +\frac{i k}{\rho h g} \epsilon_{b c}^{a} x^{b} \partial_{r}\left(J h^{-1}\left(h^{\prime}+\gamma_{0} \rho \partial_{\rho} h\right)\right) \theta^{4} \theta^{c} .
\end{aligned}
$$

The first set of (4.19-4.23) gives the following constraints:

$$
\begin{aligned}
& f g^{-1} \partial_{r}\left(J f^{-1}\left(f^{\prime}+\gamma_{0} \rho \partial_{\rho} f\right)\right)=C, \\
& g f^{-1} \partial_{t}\left(J g^{-1}\left(\dot{g}-\gamma_{4} \rho \partial_{\rho} g\right)\right)=C_{2}, \\
& (f g)^{-1}[f, g]+i k \partial_{t}\left(J g^{-1}\left(g^{\prime}+\gamma_{0} \rho \partial_{\rho} g\right)\right) \\
& \quad=(f g)^{-1}[f, g]+i k \partial_{r}\left(J f^{-1}\left(\dot{f}-\gamma_{4} \rho \partial \rho f\right)\right)=C_{3},
\end{aligned}
$$

and from the second set we obtain

$f g^{-1} \partial_{r}\left(J h^{-1}\left(h^{\prime}+\gamma_{0} \rho \partial \rho h\right)\right)=C_{4}$,

$\partial_{r}\left(J f^{-1}\left(\gamma_{4} f^{\prime}+\gamma_{0} \dot{f}\right)\right)=0$,

$(h f)^{-1}[h, f]-i k\left(J f^{-1}\left(\gamma_{4} f^{\prime}+\gamma_{0} \dot{f}\right)\right)=C_{5}$,

where the $C_{k}$ are constants.

\subsection{Solutions}

We obtained a relatively complicated set of equations, but as we are looking for static solutions we can assume that no function depends on time. In fact, the proposed choice $J^{4}=$ $0, L=2 \rho^{2} / n k$ satisfies almost all of the equations. The first nontrivial constraint is $J^{0 \prime}=0$ and it implies $J^{0}=J^{0}(\rho)$. The remaining ones are

$\gamma_{0} h+h^{\prime}+\gamma_{0} \rho \partial_{\rho} h=0$,

$J^{-1} J^{\prime}+g^{-1}\left(g^{\prime}+\gamma_{0} \rho \partial_{\rho} g\right)=0$,

$f g^{-1}\left(J f^{-1}\left(f^{\prime}+\gamma_{0} \rho \partial_{\rho} f\right)\right)^{\prime}=C$,

whereas (4.26) is satisfied identically with $C_{4}=0$. We have three equations for three frame functions $f, g$, and $h$.

When we are solving (4.29-4.31) we should not forget the diffeomorphism invariance. There are essentially two different cases. In the simplest case the frame does not depend on 
$\rho$, and the equations reduce to ordinary differential equations. We can define the radial coordinate arbitrarily, for example by fixing the frame function as $g=1$; or $h=r$; or $f g=1$. Let us briefly review these choices and the corresponding solutions.

For $g=1$, the radius $r$ is the geodesic normal coordinate and (4.29-4.31) become

$g=1, \quad J=$ const,$\quad \gamma_{0}=$ const $=\gamma$,

$f(\log f)^{\prime \prime}=$ const,$\quad \gamma+(\log h)^{\prime}=0$.

The solution is

$h=h_{0} e^{-\gamma r}, \quad f=f_{0}(\cosh 2 \beta r+1)$,

where $\beta, h_{0}$, and $f_{0}$ are the integration constants.

The corresponding classical limit is easily derived,

$$
\begin{aligned}
\mathrm{d} s^{2}= & -f^{2} \mathrm{~d} t^{2}+\mathrm{d} r^{2}+h^{2} \mathrm{~d} \Omega=-f_{0}^{2}(\cosh 2 \beta r+1)^{2} \mathrm{~d} t^{2} \\
& +\mathrm{d} r^{2}+h_{0}^{2} e^{-2 \gamma r} d \Omega,
\end{aligned}
$$

and it has the scalar curvature

$R=-8 \beta^{2}-6 \gamma^{2}+\frac{2}{h_{0}^{2}} e^{2 \gamma r}+4 \beta \frac{\beta+\gamma \sinh 2 \beta r}{\cosh ^{2} \beta r}$.

If the radius $r$ is defined such that the area of the sphere is $4 \pi r^{2}$, that is, $h=r$, we obtain

$h=r, \quad \gamma_{0}=\frac{J_{0}}{J}=-\frac{1}{r}, \quad \frac{1}{r}+(\log g)^{\prime}=0$,

$f\left(r(\log f)^{\prime}\right)^{\prime}=-\frac{C}{J_{0}} g$.

Denoting the integration constant of the third equation by $\gamma$ we find

$g=\frac{1}{\gamma r}$,

and clearly we have the same metric as (4.34) expressed in variable $\bar{r}=h_{0} e^{-\gamma r} \rightarrow r$. We further get

$f=\left(\frac{r}{h_{0}}+\frac{h_{0}}{r}\right)^{2}$

as a solution for $f$ corresponding to nonvanishing $C=$ $-8 h_{0} \mu J^{0} \gamma \neq 0$. For $C=0$ the solution is

$f=\beta r^{\alpha}$.

In particular for values $\alpha=1$ and $\beta=\gamma$ the metric has the Schwarzschild form, $f=g^{-1}$.

For the third definition of $r, f g=1$, the equations become

$f g=1, \quad(\log J g)^{\prime}=0, \quad f^{2} f^{\prime \prime}=C, \quad \gamma_{0}+(\log h)^{\prime}=0$.
We have

$g=f^{-1}, \quad J=\alpha f, \quad \log h=-\frac{J^{0}}{\alpha} \int f^{-1} \mathrm{~d} r$,

where $f$ obeys

$\frac{1}{2} \frac{\mathrm{d} f^{\prime 2}}{\mathrm{~d} f}=\frac{C}{f^{2}}$

A solution to the last equation is, for example,

$f=(\gamma r+\beta)^{p}$ for $p=1, \frac{2}{3}$,

but the integral (4.42) can be as well solved in general and yields the implicit solution

$\gamma r+\beta=\frac{2 C}{\gamma^{2}}\left(-\frac{x}{x^{2}+1}+\arctan x\right), \quad f=\frac{2 C}{\gamma^{2}} \frac{x^{2}}{x^{2}+1}$.

We have therefore in case when the metric does not depend on $\rho$ three families of solutions: essentially they represent one noncommutative space, except perhaps for singular values of the integration constants when the spaces might differ. This space is static and curved and, since everything depends on just one variable $r$, it has a straightforward classical limit.

What happens when we include the dependence on $\rho$ in the frame functions? We have seen that $\rho$ is essentially a quantum variable, so perhaps we can expect solutions which are physically more interesting. The set of (4.29-4.31) is in this case a set of partial equations in $r$ and $\rho .^{2}$ This implies that we have many more solutions, and we shall here restrict our considerations to the case when both conditions $h=r$ and $f g=1$ are fulfilled. The equation for $h$ then gives

$\gamma_{0}=-\frac{1}{r}, \quad J=-J_{0} r$

where $J_{0}$ is an arbitrary function $J_{0}=J_{0}(\rho)$ of $\rho$. Imposing $f g=1$ we see that the remaining two equations are consistent for $C=0$ and

$\left(r \partial_{r}-\rho \partial_{\rho}\right) \log f=1$.

We have already found a particular solution to this equation, $f=\gamma r$; we need therefore in addition to solve the corresponding homogeneous equation

$\left(r \partial_{r}-\rho \partial_{\rho}\right) \log F=0$,

$\overline{2}$ Note that derivatives $\partial_{\rho}$ in these equations only come from evaluating commutators with $\rho$. 
which is easy: the solution is an arbitrary function $F=$ $F(\rho r)$. Thus the general solution to (4.46) is

$f=g^{-1}=\gamma r F(\rho r)$,

with the corresponding metric

$\mathrm{d} s^{2}=-f^{2} \mathrm{~d} t^{2}+\frac{1}{f^{2}} \mathrm{~d} r^{2}+r^{2} \mathrm{~d} \Omega$.

Although the solution (4.48) is restricted in its form, in the classical limit it gives practically all static metrics, because classically $\rho$ as a constant. For example, taking

$F(x)=\sqrt{\frac{1}{x^{2}}-\frac{2 m \gamma}{x^{3}}}$

and assuming that $\rho=\gamma$ we obtain the Schwarzschild metric.

\subsection{A representation}

Noncommutative space $\mathcal{A}$ is a tensor product $\mathcal{A}=\mathcal{A}^{\prime} \otimes \mathcal{A}^{\prime \prime}$ of a first factor generated by the $\xi^{a}$ and a second factor generated by $\rho, r$, and $t$. To represent it we choose a Hilbert space $\mathcal{H}$ which is a tensor product $\mathcal{H}=\mathcal{H}^{\prime} \otimes \mathcal{H}^{\prime \prime}$, with $\mathcal{H}^{\prime}$ given by a representation $J^{a}$ of $s_{3}$ and $\mathcal{H}^{\prime \prime}=L^{2}\left(\mathbb{R}^{2}\right)$. To be explicit we consider the case defined by (4.45) and $J_{0}=1$; the commutation relations are

$[\rho, t]=i k \rho, \quad[r, t]=-i k r, \quad[\rho, r]=0$.

In this particular case the coordinates satisfy a Lie bracket relation and the corresponding Lie algebra is a singular contraction of $s_{2}(\mathbb{R})$ : the five position generators are closely related to the six generators of the Lie algebra of the Lorentz group. The $\mathcal{A}^{\prime \prime}$ can be represented, for example, on the Hilbert space $\mathcal{H}^{\prime \prime}$ of square integrable functions of two variables $(s, u)$, in analogy with the representation discussed in the previous section,

$$
\begin{aligned}
\xi^{a} \mapsto \frac{2}{n} J^{a} \otimes 1 \otimes 1, & \rho \mapsto 1 \otimes s^{-2} \otimes u, \\
t \mapsto 1 \otimes \frac{i k}{4}\left(s \frac{\mathrm{d}}{\mathrm{d} s}+\frac{\mathrm{d}}{\mathrm{d} s} s\right) \otimes 1, & r \mapsto 1 \otimes s^{2} \otimes 1 .
\end{aligned}
$$

\section{Conclusions}

We found in this paper two families of noncommutative spherically symmetric geometries which can be considered as extensions of static and cosmological solutions to the Einstein equations. The problem of extending of a classical spherically symmetric geometry to a noncommutative space (associative algebra $\mathcal{A}$ ) might seem at first sight easy. However, the simplest way of extension, the tensor product, is not satisfactory because no classically relevant metric is a product metric. Therefore one has to extend both the algebra and the associated differential calculus nontrivially, and that gives a nontrivial set of constraints.

Following the intuition that gravity is related to geometry we used a noncommutative version of the Cartan frame formalism [2]. The main input in this formalism is the set of tetrads or the frame. In the basis of the frame 1-forms the metric components are constants and the differential is defined naturally, with respect to the given or required gravitational configuration. However, the algebraic structures are rigid and there are constraints: first, the Jacobi constraints in the algebra, and second, the consistency constraints necessary for the compatibility of the algebra with the calculus.

These constraints were solved here to first order in noncommutativity. It is clearly possible to iterate and find the second and higher order equations, but it is hard to give any general statement on the existence of solutions. Another perhaps viable approach is to solve the constraints exactly, within an algebra given in advance; the problem, however, is the guessing of the initial algebra.

One of the significant features of the formalism is the possibility to generalize the diffeomorphism invariance. To first order in noncommutativity this invariance is exactly the same as in the Einstein gravity and one is allowed to choose the most convenient set of coordinates.

Our concrete problem was formulated thus: how should one extend the fuzzy sphere to a four-dimensional static or cosmological space? We first started by extending the symmetry, that is, the momentum algebra, assuming at the same time that the frame derivations are inner. This assumption gave an essentially unique solution for $\mathcal{A}$ : an algebra generated by five coordinates $\left(\zeta^{a}, r, t\right)$ or by five momenta $\left(p_{a}, \Pi, \pi_{4}\right)$ constrained by one relation, that is, possessing one Casimir operator, $\zeta^{2}$. This algebra with the associated calculus describes a spherically symmetric non-static space, which, however, is not spatially homogeneous, that is, not isotropic. Nonetheless the space has some interesting properties; for example the Hubble parameter is given by noncommutativity. Everything in the model was essentially fixed: the Jacobi constraints behaved as field equations.

In the second part of the paper we extended the original algebra by adding one variable, so $\mathcal{A}$ was generated by six coordinates ( $\left.\xi^{a}, \rho, r, t\right)$ constrained by one constraint, or by five unconstrained coordinates $\left(x^{a}, r, t\right)$. In this setup, as we have seen, one could obtain practically all static spherically symmetric configurations as classical limits. The price to pay was an additional variable $\rho$ with somewhat unusual properties: the differential calculus implied that $d \rho=0$ in the classical limit while $\rho$ was not a constant (we have for exam- 
ple $[\rho, t] \neq 0$ ). In principle one would consider the existence of an element of the algebra which is not a constant and nevertheless has vanishing differential—an undesirable feature. However, such elements are not uncommon, we mention as an example the dilatator $\Lambda$ of the quantum line. The exact role of $\rho$ in this specific case remains to be understood better, along with the question of the appropriate representation; in any case the classical relation $d \rho=0$ suggests that a natural way to interpret $\rho$ is as a Kaluza-Klein parameter which measures the internal space. In addition, one can rather easily see that in this model the momenta cannot belong to $\mathcal{A}$. Namely, solving (2.14) for $p_{\alpha}$ we obtain the solution $i k p_{a}=\delta_{a b} \xi^{b}$, $p_{0}=p_{0}(\rho, r)$, but the remaining $p_{4}=p_{4}(\rho, r, t)$ is inconsistent with the assumption $e_{4}^{a}=0$, which gives a diagonal and static metric.

Momentum operators are, in classical gravity, always external, $p_{\alpha}=e_{\alpha}^{\mu} \partial_{\mu}$. In addition when we deal with commutative space, the Jacobi identities and the de Rham consistency conditions are trivially satisfied. In the opposite, maximally constrained noncommutative case when the phase and the configuration spaces are identical, the Jacobi identities and the compatibility conditions practically fix the dynamics and the geometry. Here we observe that, with the increase of the phase space, constraints become less restrictive and we have more freedom to choose solutions: more noncommutative geometries can be defined consistently. It is certainly possible to continue along these lines and find other interesting quantum spaces with nondegenerate noncommutativity, correct symmetries, and the desired commutative limits; and that is what we plan to investigate in our future work.

Acknowledgments This work was supported by the Serbian Ministry of Education, Science and Technological Development Grant ON171031. The authors worked on the subject especially while visiting on several occasions ESI in Vienna and AEI in Berlin. They would like to take this opportunity to thank Harald Grosse, Hermann Nicolai and Stephan Theisen for their hospitality.

Open Access This article is distributed under the terms of the Creative Commons Attribution License which permits any use, distribution, and reproduction in any medium, provided the original author(s) and the source are credited.

Funded by SCOAP ${ }^{3}$ / License Version CC BY 4.0.

\section{References}

1. L.D. Faddeev, N.Y. Reshetikhin, L.A. Takhtajan, Quantization of Lie groups and Lie algebras. Leningrad Math. J. 1, 193 (1990). [Alg. Anal. 1 (1989) 178]

2. J. Madore, in An introduction to noncommutative differential geometry and its physical applications, no. 257. London Mathematical Society Lecture Note Series, 2nd edn. (Cambridge University Press, Cambridge, 1999)

3. M. Buric, T. Grammatikopoulos, J. Madore, G. Zoupanos, Gravity and the structure of noncommutative algebras. JHEP 0604, 054 (2006)

4. J. Madore, On Poisson structure and curvature. Rept. Math. Phys. 43, 231 (1999)

5. M. Cahen, S. Gutt, J. Horowitz, Homogeneous symplectic manifolds with Ricci-type curvature. J. Geom. Phys. 38, 140 (2001)

6. J. Arnlind, G. Huisken, On the geometry of Káhler-Poisson structures. arXiv:1103.5862 [math.DG]

7. J. Hoppe, Quantum theory of massless relativistic surface and a two-dimensional bound state problem. Soryushiron Kenkyu (Kyoto) 80(1989) 145, PhD Thesis, MIT, 1982

8. J. Madore, The fuzzy sphere. Class. Quant. Grav. 9, 69 (1992)

9. M. Buric, J. Madore, Spherically symmetric noncommutative space: $d=4$. Eur. Phys. J. C 58, 347 (2008)

10. M. Buric, J. Madore, Noncommutative spherical symmetry via the monopole. Int. J. Mod. Phys. A 24, 2783 (2009)

11. M. Buric, M. Wohlgenannt, Geometry of the Grosse-Wulkenhaar model. JHEP 1003, 053 (2010). [arXiv:0902.3408 [hep-th]]

12. M. Buric, J. Madore, A dynamical 2-dimensional fuzzy space. Phys. Lett. B 622, 183 (2005) 